\title{
APPROXIMATE LINEAR STABILITY ANALYSIS OF A MODEL OF ADIABATIC SHEAR BAND FORMATION*
}

BY

TIMOTHY J. BURNS

Sandia National Laboratories

\begin{abstract}
The formation of adiabatic shear bands in ductile metals under dynamic loading conditions is generally thought to result from a material instability, which is associated with a peak in the curve of engineering plastic flow stress vs. engineering shear strain. This instability arises from the effect of thermal softening, caused by irreversible adiabatic heating, which counteracts the tendency of the material to harden with increasing plastic strain. An approximate linear stability analysis of a one-dimensional rigid-thermoviscoplastic model, based on data taken from dynamic torsion experiments on thin-walled tubes of mild steel, shows that shear band formation in this situation can be interpreted as a bifurcation from a homogeneous simple shearing deformation which occurs at the peak in the homogeneous stress vs. strain curve. The asymptotic method of multiple scales is used to show that the growth rate of small perturbations on the homogeneous deformation is controlled by the ratio of the slope of the homogeneous stress vs. strain curve to the material viscosity, i.e., the rate of change of the plastic flow stress with respect to the strain-rate. In addition, it is shown that this growth rate is essentially independent of wavelength in any small perturbation. Numerical methods are used to show that this growth rate beyond the bifurcation point may not be sufficiently large for the model to account for the experimental data, and some suggestions are made on how to modify the constitutive equation so that it better fits the experimental data.
\end{abstract}

1. Introduction. A shear band is a localized region of intense plastic shearing strain, which appears in a material near a surface of maximum shear stress, during some irreversible deformation process which includes shear. The study of adiabatic shear band formation in ductile metals is of considerable interest, because these bands frequently lead to fracture, and thus to structural failure, in industrial processes which involve rapid shear, such as shaping, machining, forming, and grinding, and in military applications, such as ballistic penetration of armor and explosive fragmentation. Adiabatic shear under high pressure has also been proposed as a mechanism for producing seismic faults in the

\footnotetext{
*Received October 19, 1983. This work performed at Sandia National Laboratories supported by the U. S. Department of Energy under contract number DE-AC04-76DP00789.
} 
Earth's mantle [12] and for the impact initiation of high explosives [17]. An inclusive, critical review of adiabatic shear phenomena is given by Rogers [14], and a more recent review, including a theoretical discussion of adiabatic shear band formation, is given by Clifton [4]. Additional theoretical studies of adiabatic shear localization problems include the work of Bai [1], Burns [2], Burns \& Trucano [3], Costin, et al. [5], Dafermos \& Hsiao [6], Grady [7], Litonski [10], Merzer [11], and Pan [13]. The purpose of this paper is to analyze an idealized continuum model of controlled dynamic torsion experiments on specimens of mild, i.e., low-carbon $(<0.2 \%)$, steel, in order to study the parameters which control the growth of adiabatic shear bands in this situation, as well as to study the dynamic behavior predicted by this model.

Following Costin, Crisman, Hawley and Duffy [5], it is assumed that the dynamic flow stress $\tau$ of mild steel in shear is a function of strain $\gamma$, strain-rate $\dot{\gamma}$ and temperature $\theta$ of the form

$$
\tau(\gamma, \dot{\gamma}, \theta)=c(1-a \theta)(1+b \dot{\gamma})^{m} \gamma^{n}
$$

proposed by Litonski [10]. Values of the parameters in (1.1) for 1018 cold-rolled steel (CRS) and 1020 hot-rolled steel (HRS) have been determined from data taken in a series of torsional Kolsky (i.e., split-Hopkinson) bar simple shear experiments on thin-walled tubes of the two types of steel by Costin, et al. [5]. The constitutive equation (1.1) exhibits a competition between strain-hardening and thermal-softening, i.e., $\partial \tau / \partial \gamma>0$ and $\partial \tau / \partial \theta$ $<0$. At strain-rates which are sufficiently large for the shearing process to be adiabatic, the thermal-softening can lead to the characteristic load drop, indicated by a peak in the dynamic average engineering stress vs. strain curve of the torsion test, which has been associated with the formation of adiabatic shear bands in steel at least since the 1944 work of Zener and Hollomon [18]. Zener and Hollomon observed that, with further strain beyond the peak in the average engineering stress vs. strain curve, the macroscopically homogeneous deformation process of simple shearing must bifurcate into a more complicated heterogeneous deformation. Such a load drop and the associated formation of a single adiabatic shear band were observed by Costin, et al. [5] in their dynamic experiments on 1018 CRS, but not in similar tests on 1020 HRS, and not in quasi-static tests on either type of steel.

In this paper, an analysis will be given of the stability to small perturbations of the simple shear solution of a mathematical model of the dynamic Kolsky bar experiments of Costin, et al. on 1018 CRS. The basic assumption involved in this approach to the study of the formation of adiabatic shear bands in mild steel is that the constitutive Eq. (1.1) describes the behavior of the material even after it has begin to deform heterogeneously. Thus, local microstructural variations and small-scale temperature gradients, which certainly exist in the material during the deformation process, are assumed to be represented by small random perturbations superimposed on the homogeneous simple shearing deformation, while the phenomenological constitutive Eq. (1.1) is assumed to be independent of gradients in strain, strain-rate, or temperature. The question to be addressed, then, is whether or not the simple shearing deformation is stable to small perturbations, and, if it is not, whether the growth rates of at least some normal modes 
contained in a small perturbation are sufficiently large to account for the experimentally observed formation of adiabatic shear bands. Since the experiments described in [5] were of finite duration, the problem is thus one of determining finite-time, not asymptotic, stability.

The mathematical formulation of the Kolsky bar experiments is given in Sec. 2. In Sec. 3 , the solution corresponding to homogeneous simple shear at constant strain-rate, along with the corresponding linear equations of first variation with respect to this solution, is derived in dimensionless, scaled form, and it is shown that the linearized stability problem can be interpreted as a singular perturbation problem. An approximate analysis of this problem using the asymptotic method of multiple scales is given in Sec. 4. It is shown that the intertia term is not present in the first approximation, so that the process is dominated by viscosity, not inertia, even though the viscosity is small. It is also shown that the growth of any small perturbation in displacement or temperature on the homogeneous simple shearing deformation is essentially independent of wavelength and is controlled by the ratio of the slope of the corresponding homogeneous stress vs. strain curve to the material viscosity, i.e., $\partial \tau / \partial \dot{\gamma}$, evaluated on the homogeneous simple shearing deformation. Thus, the simple shear solution is shown to bifurcate from a stable to an unstable deformation process in the 1018 CRS at the critical strain where the homogeneous stress vs. strain curve changes from a monotone increasing to a monotone decreasing function of strain. Finally, numerical methods are used in Sec. 5 to show that this rate of growth beyond the bifurcation point is probably too small to account for the experimental data on 1018 CRS, and some suggestions are made, based in part on the analysis given in this paper, on how to modify the constitutive equation (1.1), so that it better predicts the experimental data in [5].

The analytical approach to the problem of adiabatic shear band formation taken in this paper differs from those taken in [5], [10], and [11] in several fundamental ways. First, the inertia terms are not neglected at first, as was also done in [4] and [13], so that stress equilibrium is not assumed a priori. Also, the constant initial strain-rate $\dot{\gamma}_{0}$ need not remain constant in an average sense, so that more rapid straining of one region of the tube need not be compensated for instantaneously by a less rapid straining of other regions of the tube; thus, the initially constant average strain-rate is allowed to vary as any small perturbations grow, since such a process does not violate the boundary conditions imposed by the Kolsky bar experimental apparatus. Finally, as was done in [1]-[4], [7], and [13], no specific geometrical imperfection of finite size is assumed to exist in the tube; instead, the nucleation and growth of a shear band are assumed to be governed by the amplification of random small-scale imperfections, which are no doubt present in the mechanical and thermal fields existing in the tube during the dynamic torsion process, which, in turn, is initially assumed to be homogeneous on a macroscopic scale.

The results in this paper extend those of an earlier paper [3] by explicitly accounting for the nonsteady nature of the reference homogeneous shearing solution. The conclusions of the present work differ from those in [1]-[4], and [7], which state that a specific wavelength grows the most once the deformation becomes unstable. The paper [4] treats an Arrhenius type pressure-dependent constitutive relation for the strain-rate, which is 
different from the form (1.1). The papers [6] and [7] treat a constitutive equation which is more appropriate for a fluid, in which, unlike the Litonski form given in (1.1), the shear stress is assumed to depend only on the strain-rate and temperature, and not on the strain. Such a constitutive equation cannot reproduce the experimentally measured concave average engineering stress vs. strain behavior of 1018 CRS in torsion reported by Costin, et al. [5]. The papers [2] and [3] treat mechanical constitutive equations which do not explicitly include thermal effects. Also, the interesting mathematical results in [6] are asymptotic in time, while the results presented here are asymptotic with respect to a small parameter on a finite time interval.

Conversations with L. M. Barker, R. J. Clifton, L. S. Costin, L. W. Davison, D. E. Grady, and F. R. Norwood during the course of this work are gratefully acknowledged.

2. Mathematical model. As in Litonski [10], assume that, during the dynamic torsion bar experiments of Costin, et al. [5], cross-sections normal to the axis of the thin-walled steel tube, of axial gauge length $d=2.5 \mathrm{~mm}$ and internal radius $R=0.475 \mathrm{~mm}$, undergo only irreversible stiff rotations (see Fig. 1(a), (b)), and all of the work due to this plastic torsional deformation is converted into heat. This process is then modeled as a rigid-thermoviscoplastic shearing deformation, in the Lagrangian $\bar{z}-\bar{x}$ material coordinate plane, of a thin, incompressible, semi-infinite planar sheet with mass density $\rho_{0}=8.0 \times 10^{3} \mathrm{~kg}$. $\mathrm{m}^{-3}$, as depicted in Fig. 1(c). The lower boundary is assumed to be stationary, while the upper boundary is assumed to be constrained to move at a constant velocity $v_{0}=1.25 \times$ $10^{-2} \mathrm{~km} \cdot s^{-1}$, so that the homogeneous engineering strain is given by

$$
\bar{\gamma}=\tan \Theta=\tan \left(\frac{R \phi}{d}\right),
$$

and the homogeneous strain-rate $\dot{\gamma}_{0}=v_{0} / d$ is equal to $500 \mathrm{~s}^{-1}$. It is also assumed that the sheet is so thin that the plastic flow stress $\bar{\tau}$ is uniform throughout the thickness of the sheet, i.e., $\partial \bar{\tau} / \partial \bar{y}=0$; in the experiments of Costin, et al. [5], the steel tube wall thickness

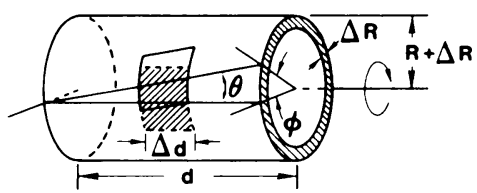

(a)

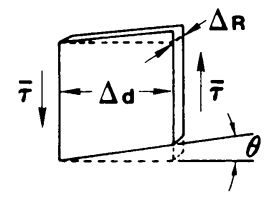

(b)

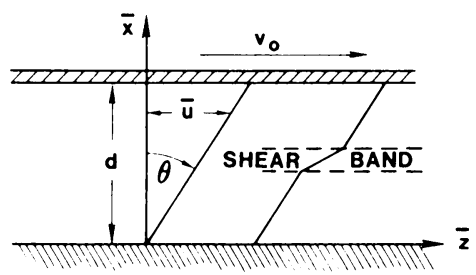

(c)

FIG. 1 
$\Delta R$ was approximately equal to $0.04 \mathrm{~mm}$. To simplify the notation in sections to follow, a bar over a variable denotes a dimensional quantity. Let $\bar{u}(\bar{x}, \bar{t})$ and $\bar{\theta}(\bar{x}, \bar{t})$ denote at time $\bar{t}$ the horizontal displacement and the absolute temperature, respectively, of the material point initially at spatial position $\bar{x}$. The engineering plastic strain and strain-rate are then defined by $\bar{\gamma}(\bar{x}, \bar{t})=\partial \bar{u}(\bar{x}, \bar{t}) / \partial \bar{x}$ and $\dot{\bar{\gamma}}(\bar{x}, \bar{t})=\partial^{2} \bar{u}(\bar{x}, \bar{t}) / \partial \bar{x} \partial \bar{t}$, respectively.

The equations governing the balance of momentum and energy for this process are then given by

$$
\begin{gathered}
\rho_{0} \frac{\partial^{2} \bar{u}}{\partial \bar{t}^{2}}=\frac{\partial \bar{\tau}}{\partial \bar{x}}, \\
\rho_{0} c_{v} \frac{\partial \bar{\theta}}{\partial \bar{t}}=\lambda \frac{\partial^{2} \bar{\theta}}{\partial \bar{x}^{2}}+\bar{\tau} \dot{\bar{\gamma}},
\end{gathered}
$$

where $\rho_{0}$ is the constant mass density of the material given above, $c_{v}=5.0 \times 10^{2} \mathrm{~J} \cdot \mathrm{kg}^{-1}$ $\cdot K^{-1}$ is its heat capacity at constant volume, and $\lambda=48 \mathrm{~J} \cdot \mathrm{m}^{-1} \cdot{ }^{-1} \cdot K^{-1}$ is its thermal conductivity in the termperature range of interest. The boundary conditions are given by

$$
\begin{aligned}
\bar{u}(0, \bar{t}) & =0, & \bar{u}(d, \bar{t})=\dot{\gamma}_{0} t, \\
\frac{\partial \bar{u}}{\partial \bar{t}}(0, \bar{t}) & =0, & \frac{\partial \bar{u}}{\partial \bar{t}}(d, \bar{t})=v_{0}, \\
\frac{\partial \bar{\theta}}{\partial \bar{x}}(0, \bar{t}) & =0, & \frac{\partial \bar{\theta}}{\partial \bar{x}}(d, \bar{t})=0,
\end{aligned}
$$

and the initial conditions are given by

$$
\bar{u}(\bar{x}, 0)=0, \quad \frac{\partial \bar{u}}{\partial \bar{t}}(\bar{x}, 0)=\dot{\gamma}_{0} \bar{x}, \quad \bar{\theta}(\bar{x}, 0)=\theta_{0},
$$

where $v_{0}$ and $\dot{\gamma}_{0}$ are the constant velocity of the upper boundary and strain-rate, respectively, which are given above. $\theta_{0}$ is the initial temperature, which, following Costin, et al. [5], is assumed to equal to the room temperature value of $300 \mathrm{~K}$. In the next section, it will be shown that the dimensionless thermal diffusivity parameter is small, so that the simple shearing process is essentially adiabatic. Adopting the results of Costin, et al. [5], the associated constitutive equation for $1018 \mathrm{CRS}$ is assumed to be given by

$$
\bar{\tau}(\bar{\gamma}, \dot{\bar{\gamma}}, \bar{\theta})=c(1-a \bar{\theta})(1+b \dot{\bar{\gamma}})^{m} \bar{\gamma}^{n},
$$

with the constant parameters

$$
\begin{aligned}
& c=6.14 \times 10^{2} \mathrm{MPa}, \\
& a=1.5 \times 10^{-3} \mathrm{~K}, \\
& b=1.0 \times 10^{3} s, \\
& m=2.5 \times 10^{-2}, \\
& n=5.0 \times 10^{-2} .
\end{aligned}
$$

These parameters were determined experimentally from Kolsky bar torsion tests which were carried out to strains which were sufficiently small that macroscopic shear localization did not occur in the samples. 
The solution to the initial-boundary value problem is given in (2.1)-(2.4) is elementary; it corresponds to homogeneous simple shear and is given by

$$
\begin{aligned}
& \bar{u}_{h}(\bar{x}, \bar{t})=\dot{\gamma}_{0} \bar{x} \bar{t}, \quad \bar{\gamma}_{h}(\bar{x}, \bar{t})=\dot{\gamma}_{0} \bar{t}, \quad \dot{\gamma}_{h}(\bar{x}, \bar{t})=\dot{\gamma}_{0}, \\
& \bar{\theta}_{h}(\bar{x}, \bar{t})=\frac{1}{a}\left\{1-\left(1-a \theta_{0}\right) \exp \left[-\frac{a c\left(1+b \dot{\gamma}_{0}\right)^{m}}{\rho_{0} c_{l}(n+1)}\left(\dot{\gamma}_{0} \bar{t}\right)^{n+1}\right]\right\},
\end{aligned}
$$

where the homogeneous temperature field was determined by the simple first-order initial value problem

$$
\rho_{0} c_{v} \frac{\partial \bar{\theta}}{\partial \bar{t}}=\bar{\tau}\left(\dot{\gamma}_{0} \bar{t}, \dot{\gamma}_{0}, \bar{\theta}(\bar{t})\right) \dot{\gamma}_{0}, \quad \bar{\theta}(0)=\theta_{0}
$$

The stability of this solution to small perturbations will be studied in the sections which follow.

3. Dimensionless, scaled variational equations. In order to use the asymptotic method of multiple scales to study the stability of the homogeneous simple shearing deformation (2.6), it is necessary to estimate the relative sizes of certain quantities. Dimensionless, scaled variables are defined by $x=\bar{x} / d, t=\dot{\gamma}_{0} \bar{t}, u=\bar{u} / d, \theta=a \bar{\theta}$, and $\tau=\bar{\tau} / c$. Introduction of these new variables into (2.1)-(2.6) leads to a nondimensionalized problem with five dimensionless groups of parameters. For $1018 \mathrm{CRS}$, these are given by

$$
\begin{aligned}
& g=a \theta_{0}=4.5 \times 10^{-1}, \\
& p=b \dot{\gamma}_{0}=5.0 \times 10^{6}, \\
& q=c /\left(\dot{\gamma}_{0}^{2} d^{2} \rho_{0}\right)=4.9 \times 10^{4}, \\
& r=\lambda /\left(\rho_{0} c_{v} \dot{\gamma}_{0} d^{2}\right)=3.8 \times 10^{-3}, \\
& s=a c /\left(\rho_{0} c_{v}\right)=2.3 \times 10^{-1} .
\end{aligned}
$$

In terms of the dimensionless variables, the constitutive equation (2.4) has the form

$$
\tau(\gamma, \dot{\gamma}, \theta)=(1-\theta)(1+p \dot{\gamma})^{m} \gamma^{n} .
$$

Also, the solution (2.6) of (2.1)-(2.4) transforms into

$$
\begin{aligned}
u_{h}(x, t) & =x t \\
\gamma_{h}(t) & =t \\
\dot{\gamma}_{h}(t) & =1 \\
\theta_{h}(t) & =1-(1-g) \exp \left[-\frac{s}{(n+1)}(1+p)^{m} t^{n+1}\right] .
\end{aligned}
$$


Note that the dimensionless time scale is equal to the time-dependent homogeneous strain, which is already dimensionless. Define the homogeneous stress $\tau_{h}$, as a function of the homogeneous strain, by

$$
\tau_{h}(t)=\tau\left(t, 1, \theta_{h}(t)\right)
$$

For notational convenience, define the four auxiliary functions

$$
\begin{array}{ll}
A(t)=\frac{\partial \tau}{\partial \theta}\left(t, 1, \theta_{h}(t)\right) & \text { (thermal-softening), } \\
B(t)=\frac{\partial \tau}{\partial \gamma}\left(t, 1, \theta_{h}(t)\right) & \text { (strain-hardening), } \\
C(t)=\frac{\partial \tau}{\partial \dot{\gamma}}\left(t, 1, \theta_{h}(t)\right) & \text { (strain-rate hardening, i.e., viscosity), } \\
f(t)=\frac{d \tau_{h}}{d t}(t) & \text { (slope of homogeneous stress vs. strain). }
\end{array}
$$

Note that, for positive values of $t, A(t)$ is negative, $B(t)$ and $C(t)$ are positive, and

$$
f(t)=B(t)+s A(t) \tau_{h}(t) .
$$

Also note that $f(t)$ changes sign from positive to negative at the critical value $t_{c}$ where the homogeneous dimensionless plastic stress (3.4) achieves a maximum with respect to the homogeneous plastic strain $t$. Plots of $\theta_{h}, \tau_{h}, A, B, C$ and $f$ as functions of $t$ are given in Figs. 2-7.

With the notation defined above, the linear equations of first variation of (2.1)-(2.4), with respect to the homogeneous simple shearing deformation (2.6), are given by

$$
\begin{aligned}
\frac{\partial^{2} \delta u}{\partial t^{2}} & =q\left[A(t) \frac{\partial \delta \theta}{\partial x}+B(t) \frac{\partial^{2} \delta u}{\partial x^{2}}+C(t) \frac{\partial^{3} \delta u}{\partial x^{2} \partial t}\right], \\
\frac{\partial \delta \theta}{\partial t} & =r \frac{\partial^{2} \delta \theta}{\partial x^{2}}+s\left[A(t) \delta \theta+B(t) \frac{\partial \delta u}{\partial x}+\left(\tau_{h}(t)+C(t)\right) \frac{\partial^{2} \delta u}{\partial x \partial t}\right]
\end{aligned}
$$

As is common in fluid dynamics stability theory (see, e.g., [9]), the stability of the deformation (3.3) to infinitesimal disturbances will be analyzed by studying the stability, to arbitrary disturbances, of the identically zero solution of the linear time-dependent boundary value problem consisting of system (3.7) together with the boundary conditions

$$
\begin{gathered}
\delta u(0, t)=\delta u(1, t)=0, \\
\frac{\partial \delta u}{\partial t}(0, t)=\frac{\partial \delta u}{\partial t}(1, t)=0, \\
\frac{\partial \delta \theta}{\partial x}(0, t)=\frac{\partial \delta \theta}{\partial x}(1, t)=0 .
\end{gathered}
$$


Thus, the stability problem of interest here involves the study of the evolution in dimensionless time of a solution of (3.7)-(3.8), with arbitrary smooth initial data satisfying the appropriate periodic boundary conditions (3.8). That is, for $0 \leqslant x \leqslant 1$,

$$
\delta u\left(x, t_{0}\right)=\zeta(x), \quad \frac{\partial \delta u}{\partial t}\left(x, t_{0}\right)=\eta(x), \quad \delta \theta\left(x, t_{0}\right)=\xi(x),
$$

where $\zeta, \eta, \xi \in C^{2}(R)$ are periodic of period $2, \zeta$ and $\eta$ are odd functions and $\xi$ is an even function of $x$, for some $t_{0}$ which is assumed to satisfy $0 \leqslant t_{0}<t_{c}$, where $t_{c}=0.16$ is the strain at which the peak in the homogeneous stress vs. strain occurs. Since the experiments of Costin, et al. [5] typically were carried out to a maximum strain of about 0.4 , the

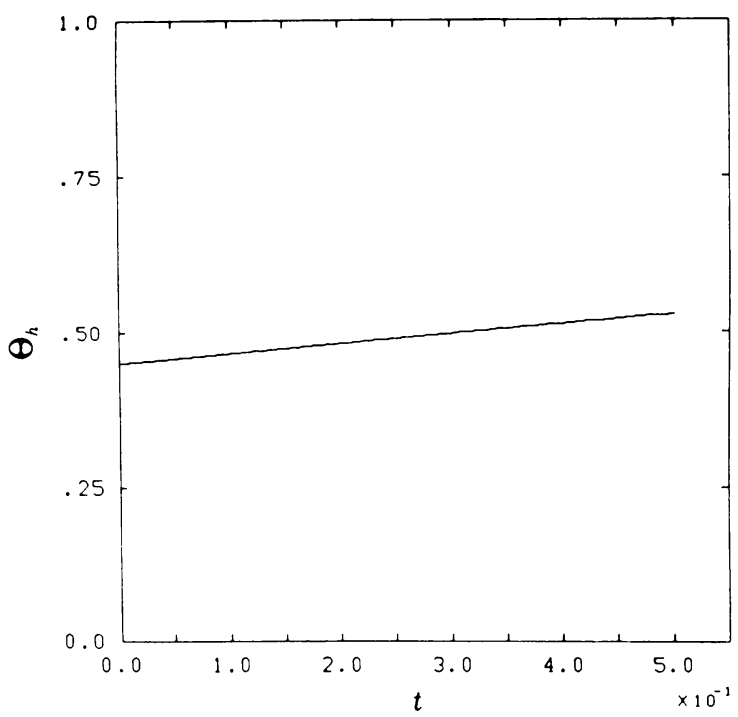

FIG. 2

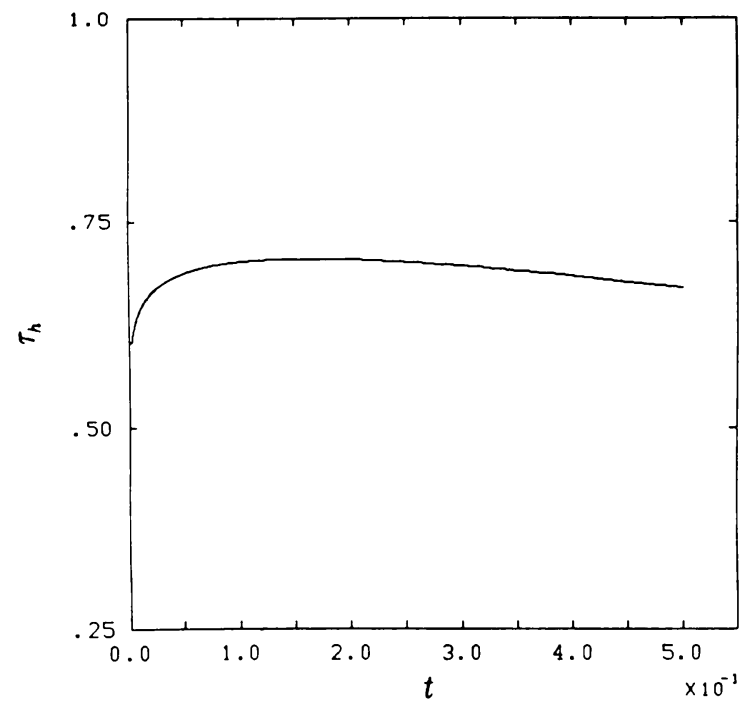

FIG. 3 
problem is not to study the asymptotic behavior of solutions of (3.7)-(3.9) as $t \rightarrow \infty$, but rather to study their behavior only on a finite interval in dimensionless time $\left[t_{0}, T\right]$, where

$$
0 \leqslant t_{0}<t_{c}<T=0.4
$$

$T$ corresponds to a dimensional time of $0.4 / 500 \mathrm{~s}^{-1}=8.0 \times 10^{-4} \mathrm{~s}$, i.e., 0.8 milliseconds. On the other hand, examination of the constant (3.1) and variable (3.4)-(3.5) coefficients in (3.7) indicates that the variational problem (3.7)-(3.9) can be treated as a problem which is asymptotic in a small parameter $\varepsilon$, i.e., asymptotic as $\varepsilon \rightarrow 0$.

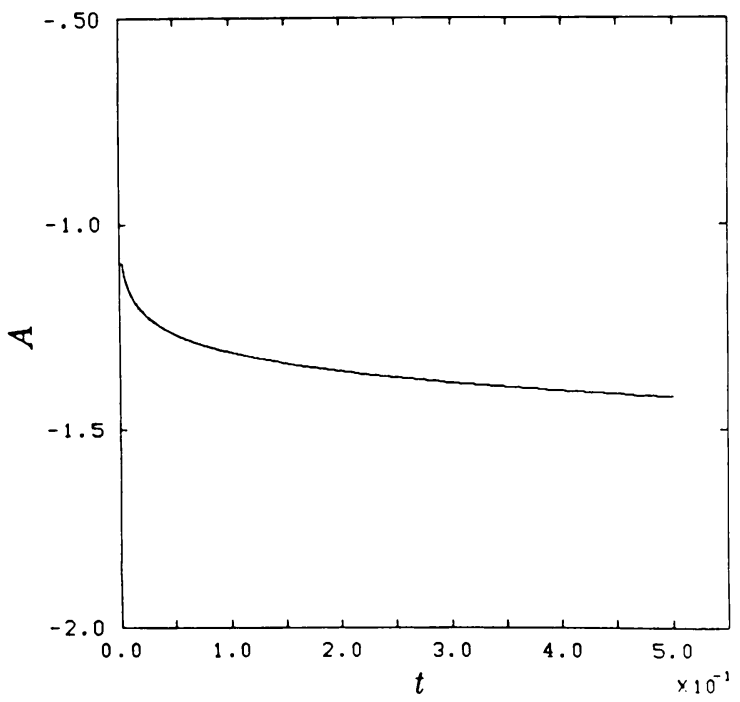

FIG. 4

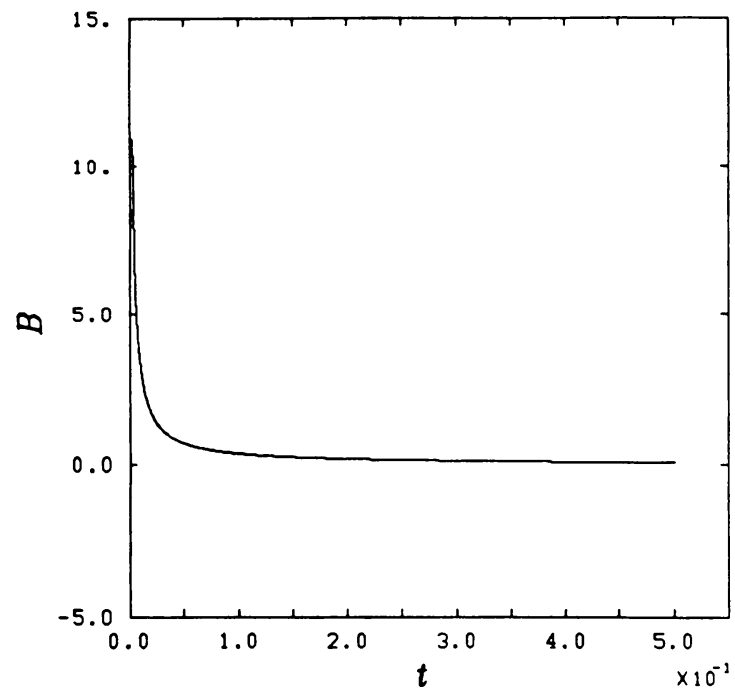

FIG. 5 
To see this, first notice that the functions $A(t), B(t), D(t),=C(t) / m, \tau_{h}(t)$ and $f(t)$ are all of the order of magnitude of 1 on $[0.0,0.4]$, as can be seen from the plots in Figs. $3-8$, except in a small neighborhood of $t=0$, where the slope of $\tau_{h}(t)$ becomes infinite. Because this singularity is clearly just an artifact of the form of the constitutive equation (1.1), it is henceforth assumed that the initial strain $t_{0}$ at which a perturbation appears on the homogeneous simple shearing deformation (2.6) is positive and sufficiently large (greater than $1.0 \times 10^{-2}$ is adequate) that $f(t)$ is of the order of magnitude of 1 on $\left[t_{0}, 0.4\right]$. (An alternative to this would be to replace $\gamma$ by $\gamma+\gamma_{0}$ in (1.1), where $\gamma_{0}$ is small

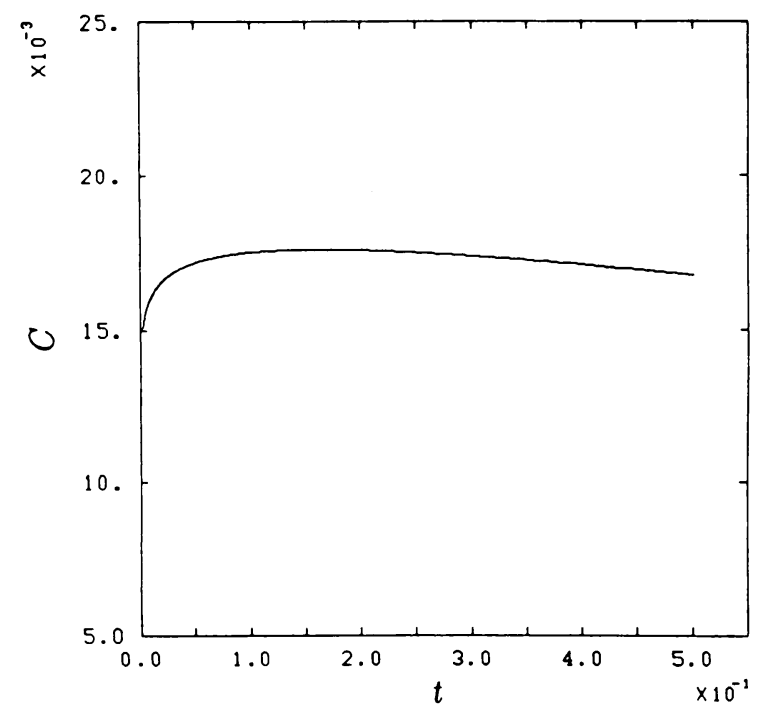

FIG. 6

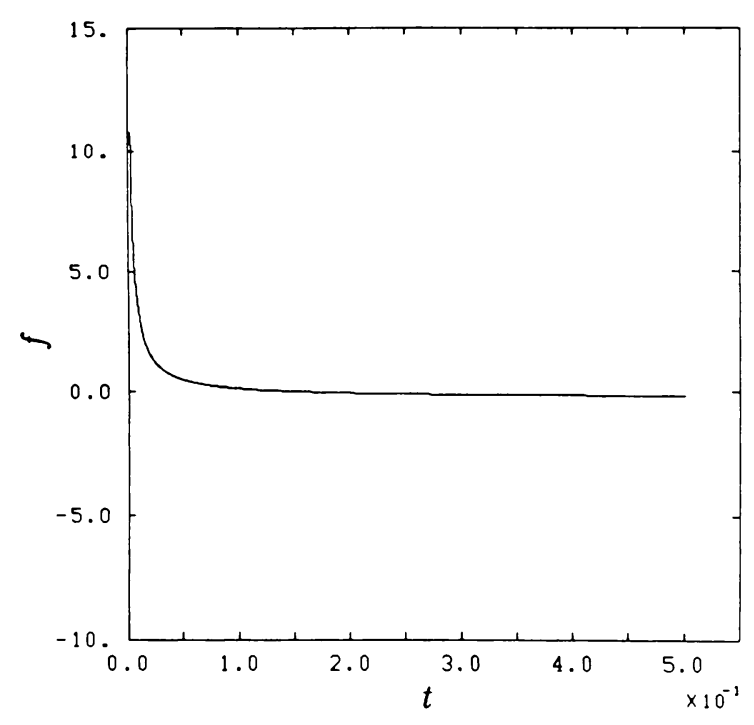

FIG. 7 
and positive, as was done by Litonski [10]). Also note that the strain-rate sensitivity parameter $m=0.025$ is much smaller than one, the thermal diffusivity parameter $r$ is approximately equal to $m^{2}, s$ is approximately of the order of magnitude of 1 , and $q^{-1}$ is approximately equal to $m^{3}$. Therefore, it seems reasonable to define $m=\varepsilon, r=\varepsilon^{2}$, $q^{-1}=\varepsilon^{3}$, and to embed (3.7) into the asymptotic problem

$$
\begin{aligned}
\varepsilon^{3} \frac{\partial^{2} \delta u}{\partial t^{2}} & =B(t) \frac{\partial^{2} \delta u}{\partial x^{2}}+\varepsilon D(t) \frac{\partial^{3} \delta u}{\partial x^{2} \partial t}+A(t) \frac{\partial \delta \theta}{\partial x}, \\
\frac{\partial \delta \theta}{\partial t} & =\varepsilon^{2} \frac{\partial^{2} \delta \theta}{\partial x^{2}}+s B(t) \frac{\partial \delta u}{\partial x}+s\left[\tau_{h}(t)+\varepsilon D(t)\right] \frac{\partial^{2} \delta u}{\partial x \partial t}+s A(t) \delta \theta,
\end{aligned}
$$

with the initial data (3.9) and boundary conditions (3.8). In the next section, two new time scales will be introduced for the system (3.10), and a uniform asymptotic expansion in $\varepsilon$ of the solution to (3.5)-(3.10) will be given, using the multiple scales method known as two-timing.

4. Two-timing. The asymptotic analysis of Eq. (3.10) can be simplified somewhat by defining a new dependent variable $\delta w(x, t)$, as follows,

$$
\delta \theta(x, t)=s \tau_{h}(t) \frac{\partial \delta u}{\partial x}(x, t)+\delta w(x, t),
$$

so that

$$
\delta w\left(x, t_{0}\right)=\xi(x)-s \tau_{h}\left(t_{0}\right) \zeta^{\prime}(x),
$$

where the prime denotes differentiation with respect to $x$, and, form (3.9),

$$
\frac{\partial \delta w}{\partial x}(0, t)=0, \quad \frac{\partial \delta w}{\partial x}(1, t)=0 .
$$

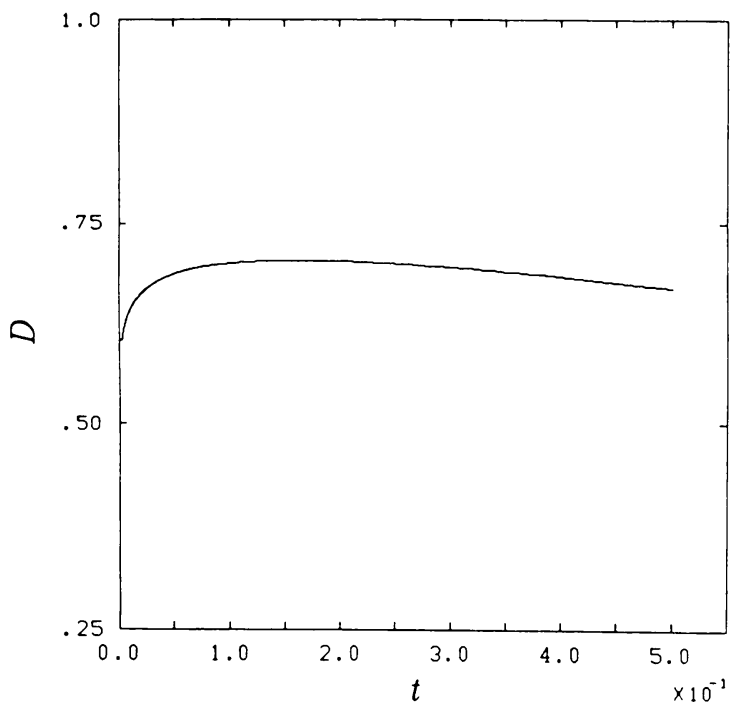

FIG. 8 
The change of variable (4.1) transforms (3.10) into the system

$$
\begin{aligned}
\varepsilon^{3} \frac{\partial^{2} \delta u}{\partial t^{2}} & =f(t) \frac{\partial^{2} \delta u}{\partial x^{2}}+\varepsilon D(t) \frac{\partial^{3} \delta u}{\partial x^{2} \partial t}+A(t) \frac{\partial \delta w}{\partial x}, \\
\frac{\partial \delta w}{\partial t} & =\varepsilon^{2}\left[\frac{\partial^{2} \delta w}{\partial x^{2}}+s \tau_{h}(t) \frac{\partial^{3} \delta u}{\partial x 3}\right]+\varepsilon s D(t) \frac{\partial^{2} \delta u}{\partial x \partial t}+s A(t) \delta w,
\end{aligned}
$$

where the definition of $f(t)$ in (3.5) and the identity (3.6) have been used to cancel several terms appearing on both sides of the equations. The initial-boundary value problem given by (3.8), (3.9), (4.2), (4.3) and (4.4) is now in suitable dimensionless, scaled form for an asymptotic analysis with respect to the small parameter $\varepsilon$ on the finite time interval $\left[t_{0}, T\right]$.

Define the two new time variables,

$$
\begin{gathered}
t^{*}=\left(t-t_{0}\right) / \varepsilon, \\
\tilde{t}=\frac{1}{\varepsilon^{2}} \int_{t_{0}}^{t} D(\rho) d \rho .
\end{gathered}
$$

Since $D(t)$ is of the order of magnitude of 1 and roughly constant, the dimensional time is approximately one microsecond $\left(1.0 \times 10^{-6} s\right)$ when $t^{*}=1$ and approximately ten nanoseconds $\left(10.0 \times 10^{-9} s\right)$ when $\tilde{t}=1$, for $\varepsilon=0.025$. Next, use (4.6) to transform the problem to depend on the faster time scale, i.e., let

$$
\delta u(x, t ; \varepsilon)=U(x, \tilde{t} ; \varepsilon), \quad \delta w(x, t ; \varepsilon)=W(x, \tilde{t} ; \varepsilon) .
$$

Since $d \tilde{t} / d t=D(t) / \varepsilon^{2}$ and $d^{2} \tilde{t} / d t^{2}=\dot{D}(t) / \varepsilon^{2}$, where the dot denotes differentiation with respect to the "slow" scaled time $t$, it follows from (4.7) that

$$
\frac{\partial \delta u}{\partial t}=\frac{D(t)}{\varepsilon^{2}} \frac{\partial U}{\partial \tilde{t}}, \quad \frac{\partial^{2} \delta u}{\partial t^{2}}=\frac{[D(t)]^{2}}{\varepsilon^{4}} \frac{\partial^{2} U}{\partial \tilde{t}^{2}}+\frac{\dot{D}(t)}{\varepsilon^{2}} \frac{\partial U}{\partial \tilde{t}},
$$

and

$$
\frac{\partial \delta w}{\partial t}=\frac{D(t)}{\varepsilon^{2}} \frac{\partial W}{\partial \tilde{t}} .
$$

It is straightforward to verify that

$$
\dot{D}(t)=\frac{p}{(1+p)} f(t)
$$

where $p$ is given in (3.1), so that $D(t)$ is of the order of magnitude of 1 on $\left[t_{0}, T\right]$. Hence, the system (4.4) transforms by (4.6) and (4.7) into

$$
\begin{aligned}
& {[D(t)]^{2}\left[\frac{\partial^{2} U}{\partial \tilde{t}^{2}}-\frac{\partial^{3} U}{\partial x^{2} \partial \tilde{t}}\right]=\varepsilon\left[f(t) \frac{\partial^{2} U}{\partial x^{2}}+A(t) \frac{\partial W}{\partial x}\right]-\varepsilon^{2} \dot{D}(t) \frac{\partial U}{\partial \tilde{t}},} \\
& D(t) \frac{\partial W}{\partial \tilde{t}}=\varepsilon s[D(t)]^{2} \frac{\partial^{2} U}{\partial x \partial \tilde{t}}+\varepsilon^{2} s A(t) W+\varepsilon^{4}\left[\frac{\partial^{2} W}{\partial x^{2}}+s \tau_{h}(t) \frac{\partial^{3} U}{\partial x^{3}}\right] .
\end{aligned}
$$

For notational convenience, define new coefficient functions by

$$
\hat{A}\left(t^{*}\right)=A\left(t_{0}+\varepsilon t^{*}\right), \quad \hat{B}\left(t^{*}\right)=B\left(t_{0}+\varepsilon t^{*}\right), \ldots
$$


Then (4.8) can be rewritten in terms of the two time scales (4.5) and (4.6) as follows.

$$
\begin{gathered}
{\left[\hat{D}\left(t^{*}\right)\right]^{2}\left[\frac{\partial^{2} U}{\partial \tilde{t}^{2}} \frac{\partial^{3} U}{\partial x^{2} \partial \tilde{t}}\right]=\varepsilon\left[\hat{f}\left(t^{*}\right) \frac{\partial^{2} U}{\partial x^{2}}+\hat{A}\left(t^{*}\right) \frac{\partial W}{\partial x}\right]-\varepsilon^{2} \hat{\dot{D}}\left(t^{*}\right) \frac{\partial U}{\partial \tilde{t}},} \\
\hat{D}\left(t^{*}\right) \frac{\partial W}{\partial \tilde{t}}=\varepsilon s\left[\hat{D}\left(t^{*}\right)\right]^{2} \frac{\partial^{2} U}{\partial x \partial \hat{t}}+\varepsilon^{2} s \hat{A}\left(t^{*}\right) W+\varepsilon^{4}\left[\frac{\partial^{2} W}{\partial x^{2}}+s \hat{\tau}_{h}\left(t^{*}\right) \frac{\partial^{3} U}{\partial x^{3}}\right] .
\end{gathered}
$$

Following Kevorkian and Cole [8], a solution of (4.9), and hence of (4.4), with appropriate initial and boundary conditions, is assumed to have the two time-variable uniform asymptotic expansion

$$
\begin{aligned}
& \delta u(x, t ; \varepsilon)=U(x, \tilde{t} ; \varepsilon)=\sum_{n=0}^{N} F_{n}\left(x, \tilde{t}, t^{*}\right) \varepsilon^{n}+O\left(\varepsilon^{N+1}\right), \\
& \delta w(x, t ; \varepsilon)=W(x, \tilde{t} ; \varepsilon)=\sum_{n=0}^{N} G_{n}\left(x, \tilde{t}, t^{*}\right) \varepsilon^{n}+O\left(\varepsilon^{N+1}\right) .
\end{aligned}
$$

From (4.5), (4.6), and (4.10), it follows that

$$
\begin{aligned}
\frac{\partial \delta u}{\partial t}= & {\left[\frac{\partial F_{0}}{\partial \tilde{t}}+\varepsilon \frac{\partial F_{1}}{\partial \tilde{t}}+\varepsilon^{2} \frac{\partial F_{2}}{\partial \tilde{t}}+\cdots\right] \frac{d \tilde{t}}{d t} } \\
& +\left[\frac{\partial F_{0}}{\partial t^{*}}+\varepsilon \frac{\partial F_{1}}{\partial t^{*}}+\varepsilon^{2} \frac{\partial F_{2}}{\partial t^{*}}+\cdots\right] \frac{d t^{*}}{d t} \\
= & \frac{D(t)}{\varepsilon^{2}} \frac{\partial F_{0}}{\partial \tilde{t}}+\frac{1}{\varepsilon}\left[D(t) \frac{\partial F_{1}}{\partial \tilde{t}}+\frac{\partial F_{0}}{\partial t^{*}}\right]+\left[D(t) \frac{\partial F_{2}}{\partial \tilde{t}}+\frac{\partial F_{1}}{\partial t^{*}}\right] \\
& +\varepsilon\left[D(t) \frac{\partial F_{3}}{\partial \tilde{t}}+\frac{\partial F_{2}}{\partial t^{*}}\right]+\varepsilon^{2}\left[D(t) \frac{\partial F_{4}}{\partial \tilde{t}}+\frac{\partial F_{3}}{\partial t^{*}}\right]+\cdots
\end{aligned}
$$

Consequently, from (3.9) and (4.2), the only nonzero initial conditions for the terms in the expansions in (4.10) and (4.11) are given by

$$
\begin{aligned}
F_{0}(x, 0,0) & =\zeta(x), \\
D\left(t_{0}\right) \frac{\partial F_{2}}{\partial \tilde{t}}(x, 0,0)+\frac{\partial F_{1}}{\partial t^{*}}(x, 0,0) & =\eta(x), \\
G_{0}(x, 0,0) & =\xi(x)-s \tau_{h}\left(t_{0}\right) \zeta^{\prime}(x) .
\end{aligned}
$$

Similarly, the boundary conditions (3.8) and (4.3) require that

$$
\begin{aligned}
F_{n}\left(0, \tilde{t}, t^{*}\right) & =F_{n}\left(1, \tilde{t}, t^{*}\right)=0, \quad n=0,1, \ldots, \\
\frac{\partial G_{n}}{\partial x}\left(0, \tilde{t}, t^{*}\right) & =\frac{\partial G_{n}}{\partial x}\left(1, \tilde{t}, t^{*}\right)=0, \quad n=0,1, \ldots,
\end{aligned}
$$

as well as that each term in the expansion (4.11) vanish at $x=0$ and $x=1$. To use (4.10) to find an approximate solution of the stability problem, the expressions need to be differentiated with respect to the fastest time variable $\tilde{t}$. Using (4.5) and (4.6), it follows that

$$
d t^{*} / d \tilde{t}=\varepsilon / \hat{D}\left(t^{*}\right) .
$$


Hence,

$$
\begin{aligned}
\frac{\partial U}{\partial \tilde{t}} & =\frac{\partial}{\partial \tilde{t}}\left[F_{0}+\varepsilon F_{1}+\varepsilon^{2} F_{2}+\cdots\right] \\
& =\left[\frac{\partial F_{0}}{\partial \tilde{t}}+\frac{\varepsilon}{\hat{D}} \frac{\partial F_{0}}{\partial t^{*}}\right]+\varepsilon\left[\frac{\partial F_{1}}{\partial \tilde{t}}+\frac{\varepsilon}{\hat{D}} \frac{\partial F_{1}}{\partial t^{*}}\right]+\varepsilon^{2}\left[\frac{\partial F_{2}}{\partial \tilde{t}}+\frac{\varepsilon}{\hat{D}} \frac{\partial F_{2}}{\partial t^{*}}\right]+\cdots \\
& =\frac{\partial F_{0}}{\partial \tilde{t}}+\varepsilon\left[\frac{\partial F_{1}}{\partial \tilde{t}}+\frac{1}{\hat{D}} \frac{\partial F_{0}}{\partial t^{*}}\right]+\varepsilon^{2}\left[\frac{\partial F_{2}}{\partial \tilde{t}}+\frac{1}{\hat{D}} \frac{\partial F_{1}}{\partial t^{*}}\right]+\cdots,
\end{aligned}
$$

and

$$
\begin{aligned}
\frac{\partial^{2} U}{\partial \tilde{t}^{2}}= & \frac{\partial^{2} F_{0}}{\partial \tilde{t}^{2}}+\varepsilon\left[\frac{\partial^{2} F_{1}}{\partial \tilde{t}^{2}}+\frac{2}{\hat{D}} \frac{\partial^{2} F_{0}}{\partial \tilde{t} \partial t^{*}}\right] \\
& +\varepsilon^{2}\left[\frac{\partial^{2} F_{2}}{\partial \tilde{t}^{2}}+\frac{2}{\hat{D}} \frac{\partial^{2} F_{1}}{\partial \tilde{t} \partial t^{*}}+\frac{1}{\hat{D}^{2}} \frac{\partial^{2} F_{0}}{\partial t^{* 2}}\right]+\cdots
\end{aligned}
$$

Similarly,

$$
\frac{\partial W}{\partial \tilde{t}}=\frac{\partial G_{0}}{\partial \tilde{t}}+\varepsilon\left[\frac{\partial G_{1}}{\partial \tilde{t}}+\frac{1}{\hat{D}} \frac{\partial G_{0}}{\partial t^{*}}\right]+\varepsilon^{2}\left[\frac{\partial G_{2}}{\partial \tilde{t}}+\frac{1}{\hat{D}} \frac{\partial G_{1}}{\partial t^{*}}\right]+\cdots
$$

Substituting (4.14)-(4.16) into (4.9) and collecting terms of the same order of magnitude in $\varepsilon$ leads to the following system of partial differential equations for the terms in the expansions (4.10).

In the expansion for $\delta u$, the first two equations are given by

$$
\begin{gathered}
\hat{D}^{2}\left[\frac{\partial^{2} F_{0}}{\partial \tilde{t}^{2}}-\frac{\partial^{3} F_{0}}{\partial x^{2} \partial \tilde{t}}\right]=0 \\
\hat{D}^{2}\left[\frac{\partial^{2} F_{1}}{\partial \tilde{t}^{2}}-\frac{\partial^{3} F_{1}}{\partial x^{2} \partial \tilde{t}}\right]+\hat{D}\left[2 \frac{\partial^{2} F_{0}}{\partial \tilde{t} \partial t^{*}}-\frac{\partial^{3} F_{0}}{\partial x^{2} \partial t^{*}}\right]=\hat{f} \frac{\partial^{2} F_{0}}{\partial x^{2}}+\hat{A} \frac{\partial G_{0}}{\partial x},
\end{gathered}
$$

and, for $\delta w$,

$$
\begin{gathered}
\hat{D} \frac{\partial G_{0}}{\partial \tilde{t}}=0 \\
\hat{D} \frac{\partial G_{1}}{\partial \tilde{t}}+\frac{\partial G_{0}}{\partial t^{*}}=s \hat{D}^{2} \frac{\partial^{2} F_{0}}{\partial x \partial \tilde{t}} .
\end{gathered}
$$

These four equations are sufficient to determine the first approximations $F_{0}$ and $G_{0}$. First, (4.19) implies that $G_{0}$ is independent of $\tilde{t}$, so that $G_{0}=c_{0}\left(x, t^{*}\right)$. By (4.20),

$$
\hat{D} \frac{\partial}{\partial \tilde{t}}\left[G_{1}-s \hat{D} \frac{\partial F_{0}}{\partial x}\right]=-\frac{\partial c_{0}}{\partial t^{*}}
$$

Following Kevorkian and Cole [8], by requiring that no secular terms in $\tilde{t}$ appear in the expression for $G_{1}, G_{0}$ must also be independent of $t^{*}$. Hence, $G_{0}$ is determined only by its initial data, so that, by (4.12),

$$
G_{0}\left(x, \tilde{t}, t^{*}\right)=\xi(x)-s \tau_{h}\left(t_{0}\right) \zeta^{\prime}(x) .
$$


Next, (4.17) implies that $\partial F_{0} / \partial \tilde{t}$ satisfies a homogeneous diffusion equation on the fast $\tilde{t}$ scale. This equation is equivalent to the nonhomogeneous diffusion equation for $F_{0}$,

$$
\frac{\partial F_{0}}{\partial \tilde{t}}-\frac{\partial^{2} F_{0}}{\partial x^{2}}=a_{0}\left(x, t^{*}\right)
$$

where the function $a_{0}$ must still be determined. The initial data (4.12) imply that $\partial F_{0}(x, 0,0) / \partial \tilde{t}=0$, so it follows that $F_{0}$ is independent of $\tilde{t}$, and the solution to (4.22) is given by the constant (on the $\tilde{t}$ time scale) forced solution of the ordinary differential equation

$$
-\frac{\partial^{2} F_{0}}{\partial x^{2}}=a_{0}\left(x, t^{*}\right)
$$

Finally, examination of (4.18) and the fact that $F_{0}$ is independent of $\tilde{t}$, along with the requirement that there be no secular terms in $\tilde{t}$ present in the expression for $F_{1}$, leads to an ordinary differential equation for the determination of $a_{0}\left(x, t^{*}\right)$, and thus for $F_{0}$, given by

$$
\frac{\partial a_{0}}{\partial t^{*}}+\frac{\hat{f}\left(t^{*}\right)}{\hat{D}\left(t^{*}\right)} a_{0}=\frac{\hat{A}\left(t^{*}\right)}{\hat{D}\left(t^{*}\right)} \frac{\partial G_{0}}{\partial x},
$$

so that $F_{0}$ satisfies the nonhomogeneous turning-point equation

$$
\frac{\partial F_{0}}{\partial t^{*}}+\frac{\hat{f}\left(t^{*}\right)}{\hat{D}\left(t^{*}\right)} F_{0}=-\frac{\hat{A}\left(t^{*}\right)}{\hat{D}\left(t^{*}\right)} \int_{0}^{x} G_{0}(y, 0,0) d y .
$$

Using the initial data once more, it follows that

$$
\begin{aligned}
F_{0}\left(x, \tilde{t}, t^{*}\right)= & \zeta(x) \exp \left[-\int_{0}^{t^{*}} \frac{\hat{f}(\rho)}{\hat{D}(\rho)} d \rho\right] \\
& -\left[\int_{0}^{x} \xi(y) d y-s \tau_{h}\left(t_{0}\right) \zeta(x)\right] \int_{0}^{t^{*}} \exp \left[-\int_{\sigma}^{t^{*}} \frac{\hat{f}(\rho)}{\hat{D}(\rho)} d \rho\right] \frac{\hat{A}(\sigma)}{\hat{D}(\sigma)} d \sigma .
\end{aligned}
$$

Transforming this expression to one in $t$ instead of $t^{*}$ by using (4.5) and setting $\varepsilon=m$, it follows that, to a first approximation, a small perturbation in displacement on the homogeneous simple shearing deformation grows, with increasing dimensionless time $t$, according to the formula

$$
\begin{aligned}
\delta u(x, t)= & \zeta(x) \exp \left[-\frac{1}{m} \int_{t_{0}}^{t} \frac{f(\alpha)}{D(\alpha)} d \alpha\right] \\
& -\frac{1}{m}\left[\int_{0}^{x} \xi(y) d y-s \tau_{h}\left(t_{0}\right) \zeta(x)\right] \\
& \times \int_{t_{0}}^{t} \exp \left[-\frac{1}{m} \int_{\beta}^{t} \frac{f(\alpha)}{D(\alpha)} d \alpha\right] \frac{A(\beta)}{D(\beta)} d \beta .
\end{aligned}
$$


By (4.1), therefore, a related approximate formula for the growth in time of a small temperature perturbation can be determined. It is given by

$$
\begin{aligned}
\delta \theta(x, t)= & s \tau_{h}(t)\left\{\zeta^{\prime}(x) \exp \left[-\frac{1}{m} \int_{t_{0}}^{t} \frac{f(\alpha)}{D(\alpha)} d \alpha\right]-\frac{1}{m}\left[\xi(x)-s \tau_{h}\left(t_{0}\right) \zeta^{\prime}(x)\right]\right. \\
& \left.\times \int_{t_{0}}^{t} \exp \left[-\frac{1}{m} \int_{\beta}^{t} \frac{f(\alpha)}{D(\alpha)} d \alpha\right] \frac{A(\beta)}{D(\beta)} d \beta\right\} \\
& +\left[\xi(x)-s \tau_{h}\left(t_{0}\right) \zeta^{\prime}(x)\right] .
\end{aligned}
$$

The approximation procedure can be carried out to higher orders in $\varepsilon$, but it is not necessary to do so for the purposes of this study.

5. Discussion. The two dimensionless time scales (4.5) and (4.6) could be referred to as the viscosity and inertia scales, respectively. The expressions (4.24) and (4.25), which were derived in the last section, show that, in the first approximation, the growth of small perturbations in displacement or temperature on the homogeneous simple shearing deformation (2.6) takes place on the viscosity time-scale, while a small perturbation in velocity does not change with time. Another way of stating this is to say that the inertia term does not appear in the equations of first approximation, so that the process is viscosity-dominated, not inertia-dominated. Also, the rate of growth of small perturbations is seen from (4.24) and (4.25) to be controlled by the area under the curve of $-f(t) / C(t)$, where $C(t)=m D(t)$, which is the ratio of the slope of the homogeneous stress vs. strain curve to the viscosity, or strain-rate sensitivity, of the material. Since $C(t)$ is approximately constant, it follows that the slope of the homogeneous stress vs. strain curve, $f(t)$, is the dominant parameter controlling this growth rate. This agrees with the results of Burns [2] and Pan [13] for strictly mechanical processes. It is also clear that, in (4.24) and (4.25), the term

$$
\exp \left[-\frac{1}{m} \int_{t_{0}}^{t} \frac{f(\alpha)}{D(\alpha)} d \alpha\right]
$$

must decay exponentially for values of the plastic strain less than the critical value $t_{c}$ at which the peak in the $\tau_{h}(t)$ curve occurs. Once this critical value is exceeded, the term begins to grow exponentially, so that exponential growth in dimensionless time $t$ occurs in both (4.24) and (4.25) for $t>t_{c}$. This follows from splitting the integrals in (4.24) and (4.25) up into sums of integrals on $\left[t_{0}, t_{c}\right]$ and $\left[t_{c}, t\right]$ for $t>t_{c}$; the integral on the first subinterval remains constant, while that on the second subinterval grows exponentially at a rate which depends on how rapidly $f(t)$ decreases.

Examination of the form in which the initial data appear in the explicit expressions (4.24) and (4.25) indicates that no Fourier mode is selected for more rapid growth or decay. This is not surprising, because it was shown that the differential equation (4.23) which determines $F_{0}$ is an ordinary differential equation. There is, however, some smoothing by integration of higher frequencies in the initial thermal perturbation on the displacement perturbation growth, while the initial displacement perturbation appears in 
terms of its derivative in the expression for thermal perturbation growth, so that the effect of higher frequencies can be amplified. This does not affect the rate of growth, however; it only modifies the initial amplitude of a specific Fourier mode.

In the experiments of Costin, et al. [5], the final strain distribution in a tube after loading was found by measuring the inclination of a fine line which had been scribed parallel to the tube axis on the inner wall of the tube during preparation of the sample. Thus, the final displacement of an initially vertical line in the simple shearing deformation in Fig. 1(c) corresponds to the inclination of the scribe line. For the homogeneous simple

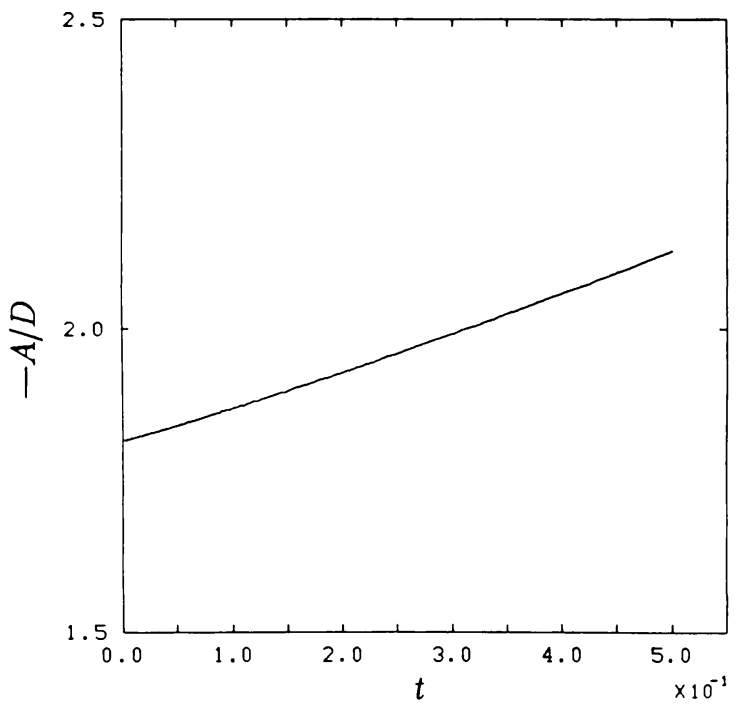

Fig. 9

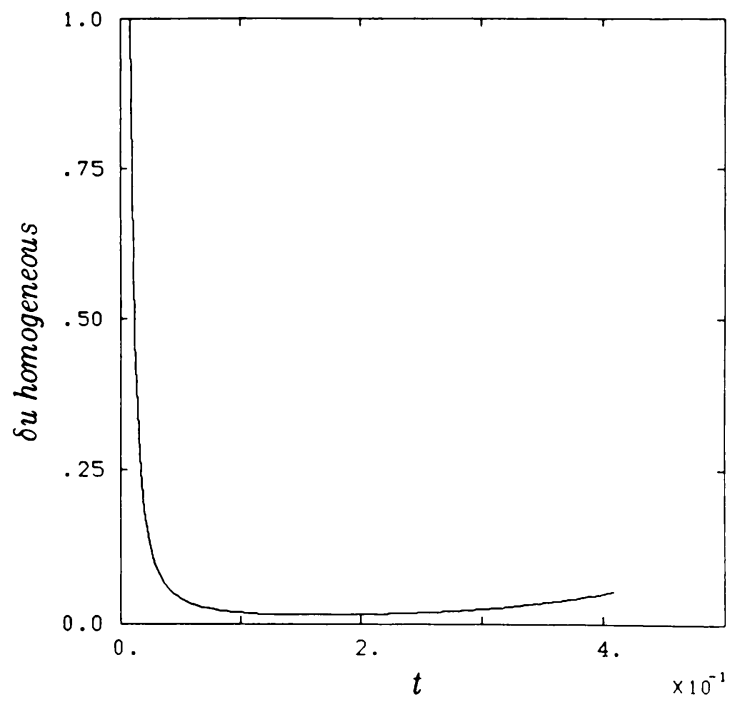

FIG. 10 
shearing deformation (2.6), which corresponds to an experiment in which no shear band was observed to form, this inclination was uniform with a constant angle to the vertical. For a nonhomogeneous deformation in which a single shear band formed, there was a much a larger inclination in the shear band region, as indicated in Fig. 1(c). For example, as reported by Costin, et al. [5], post-test measurements on specimens of $1018 \mathrm{CRS}$ for which the total average strain was approximately 0.3 indicated that the material outside the shear band had deformed to a strain of about 0.1 to 0.2 , whereas within the shear band, which covered about $15 \%$ or $20 \%$ of the length of the specimen, the shear angle $\Theta$

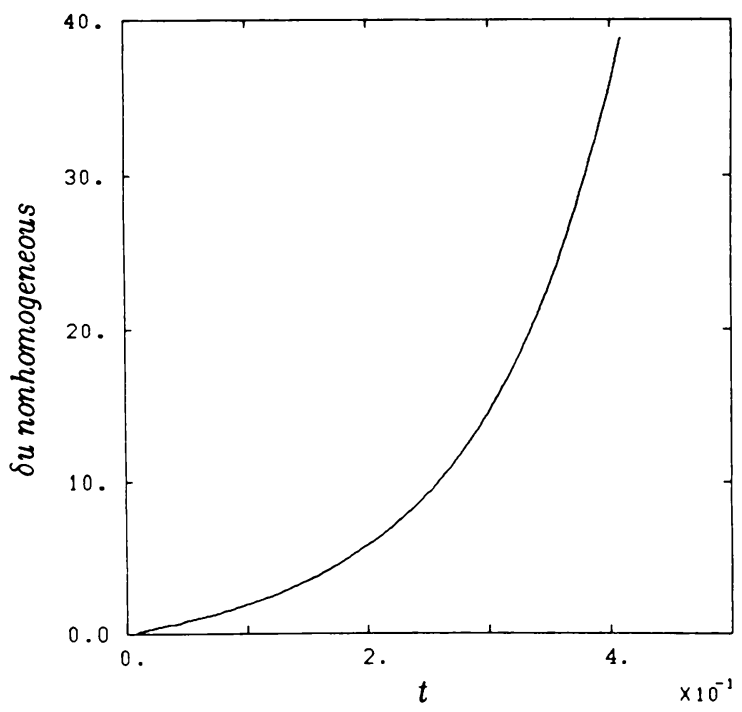

FIG. 11

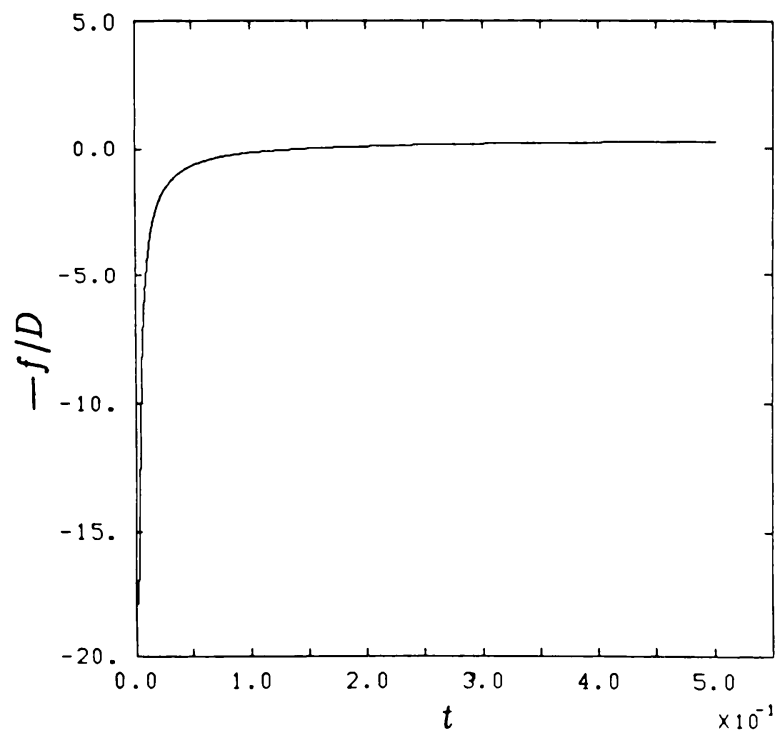

Fig. 12 
lay in the range 0.7 to 1.0. In order to estimate whether the growth of a small perturbation in displacement, as predicted by (4.24), would predict the observed experimental outcome, two numerical calculations of (4.23) were performed. Because perturbations were assumed to be arbitrary in the analysis above, it was decided to determine how (4.24) would amplify a small perturbation by numerical integration of (4.23) on $\left[t_{0}, T\right]$ as follows. First, the equation was integrated with initial value one and with the thermal forcing term, i.e., the right-hand side of (4.23), set equal to zero. Then, the equation was integrated again, this time with initial value equal to zero and with the forcing term set equal to $-A(t) / D(t)$, which is plotted in Fig. 9. An appropriate linear combination of the results of the two integrations then gives the value of (4.24) at time $T$ for specified values of the initial perturbations. The integrations were performed on a CRAY-1 computer, using a version of the $D E / S T E P / I N T R P$ package of Adams codes of Shampine and Gordon [15]. The results are presented in Fig. 10 and 11. As can be seen from the figures, the total growth of a small perturbation in displacement or temperature, with initial amplitude, say of the order of magnitude of $\varepsilon^{2}$ with $\varepsilon=0.025$, is of the order of magnitude of $\varepsilon$. This is insufficient to account for the experimentally observed formation of a shear band when added to the displacement predicted by the reference homogeneous simple shearing solution (3.3).

Examination of the slope $f(t)$ of $\tau_{h}(t)$, given in Fig. 7, or of the behavior of the function $-f(t) / D(t)$ given in Fig. 12, indicates that the small total perturbation growth results from the fact that the homogeneous stress decreases very slowly with increasing strain after the peak in the stress has been exceeded. According to the analysis in Sec. 4, it follows that one way to modify the constitutive equation (1.1) to force more rapid perturbation growth is to have more rapid thermal softening, so that $f(t)$ decreases more rapidly beyond the peak in $\tau_{h}(t)$, in a way that (1.1), which has been fit experimentally for relatively small values of the shear strain, is still valid for small strains. Another possible modification of (1.1) is suggested by the work of Pan [13] and the references he cites in his paper. The idea would be to allow the strain-rate sensitivity $C(t)$, i.e., the viscosity, to decrease substantially with increasing strain, and thus with increasing temperature, as occurs in many fluids (see, e.g., [16]). As shown by Pan, even small viscosity can significantly retard the rate of localization of an originally homogeneous simple shearing deformation, so that a substantial reduction in viscosity could have a significant effect on the rate of unstable perturbation growth. Such a modification will be discussed in a subsequent paper.

\section{REFERENCES}

[1] Y. L. Bai, Thermo-plastic instability in simple shear, J. Mech. Phys. Solids 30, 195-207 (1982)

[2] T. J. Burns, Dynamic instability of a homogeneous deformation of a thin elastic bar, Quart. Appl. Math. 40 , 357-361 (1982)

[3] T. J. Burns and T. G. Trucano, Instability in simple shear deformations of strain-softening materials, Mech. Mat. 1, 313-324 (1982)

[4] R. J. Clifton, Adiabatic shear banding, in Materials response to ultra-high loading rates, NMAB-356, National Materials Advisory Board (NRC), Washiangton, D.C., 1980, Chap. 8

[5] L. S. Costin, E. E. Crisman, R. H. Hawley, and J. Duffy, On the localisation of plastic flow in mild steel tubes under dynamic torsional loading, in Proc. 2 nd conf. on mechanical properties of materials at high rates of strain, (J. Harding, ed.) Inst. Phys. Conf. Ser. No. 47: Chap. 1, p. 90, Adam Hilger, Bristol, 1979 
[6] C. M. Dafermos and L. Hsiao, Adiabatic shearing of incompressible fluids with temperature-dependent viscosity, Quart. Appl. Math. 41, 45-58 (1983)

[7] D. E. Grady, Shock deformation of brittle solids, J. Geophys. Res. (B2) 85, 913-924 (1980)

[8] J. Kevorkian and J. D. Cole, Perturbation methods in applied mathematics, Springer-Verlag, New York, 1981, Chap. 3

[9] L. D. Landau and E. M. Lifschitz, Fluid mechanics, Pergamon Press, Oxford, 1959, Sec. 27

[10] J. Litonski, Plastic flow of a tube under adiabatic torsion, Bull. l'Academie Polonaise des Sciences 25, 7-14 (1977)

[11] A. M. Merzer, Modelling of adiabatic shear band development from small imperfections, J. Mech. Phys. Solids 30, 323-338 (1982)

[12] E. Orowan, Mechanism of seismic faulting, in Rock deformation (a symposium), D. Griggs \& J. Handin, ed., Geological Society of American Memoir 79, Waverly Press, Baltimore, 1960, Chap. 12

[13] J. Pan, Perturbation analysis of shear strain localization in rate sensitive materials, Int. J. Solids Structures 19, 153-164 (1983)

[14] H. C. Rogers, Adiabatic plastic deformation, Ann. Rev. Mater. Sci. 9, 283-311 (1979)

[15] L. F. Shampine and M. K. Gordon, Computer solution of ordinary differential equations-the initial value problem, W. H. Freeman \& Co., San Francisco, 1975

[16] D. Tabor, Gases, liquids and solids, 2nd ed., Cambridge University Press, Cambridge, 1979, Chap. 11

[17] R. E. Winter and J. E. Field, The role of localized plastic flow in the impact initiation of explosives, Proc. R. Soc. Lond. A343, 399-413 (1975)

[18] C. Zener and J. H. Hollomon, Effect of strain rate upon plastic flow of steel, J. Appl. Phys. 15, 22-32 (1944) 